\title{
NORMOGRAMA DE PREDICCIÓN DE LA FLUIDEZ VERBAL A TRAVÉS DE VARIABLES DE INTERÉS NEUROCLÍNICO
}

\section{Predictive Nomogram OF VeRBAL FLUIDITY THROUGH VARIABLES OF NEUROCLINICAL INTEREST}

\author{
Gema Arévalo Arévalo, Dr. José Vte Carmona Simarro \\ 1 ORCID: https://orcid.org/0000-0002-5838-2035 \\ Universidad Cardenal Herrera CEU Universities \\ 2 Universidad Cardenal Herrera CEU Universities
}

Arévalo Arévalo, G., \& Carmona Simarro, J. (2020). NORMOGRAMA DE PREDICCIÓN DE LA FLUIDEZ VERBAL A TRAVÉS DE VARIABLES DE INTERÉS NEUROCLÍNICO. Revista Ene De Enfermería, 14(1). Consultado de http://www.ene-enfermeria.org/ojs/index.php/ENE/article/view/969 


\section{Resumen}

El aumento en la esperanza de vida ha supuesto el envejecimiento progresivo de la población y un aumento en la prevalencia de enfermedades neurodegenerativas. La degeneración cerebral puede iniciarse en individuos de mediana edad y dado que en ausencia de patología neurodegenerativa existe una pérdida progresiva de sinapsis durante la neurosenescencia, la detección precoz de algún estado predemencial por medio de test neuropsicológicos de Fluidez Verbal (FV) podría evitar que la mayoría de los casos de demencia fueran de aparición esporádica.

Los principales procesos degenerativos corticales de aparición presenil, afectan a áreas cerebrales específicas originando alteraciones lingüísticas como la afasia semántica - vinculada con la integridad de los lóbulos temporales - o fonológica - vinculada con la integridad de los lóbulos frontales-. La FV declina con la edad, pero no se conoce con exactitud si los patrones de deterioro son cualitativamente diferentes de los observados en el proceso de envejecimiento normal.

La muestra estaba compuesta por 118 sujetos: $34.7 \%$ hombres y el $65.3 \%$ mujeres, con una media de edad de $52.95 \pm 18.87$. Se analizó la relación de la edad con pruebas de FV semántica y fonológica, y de la relación de las pruebas de FV entre sí. De esta manera se estableció un modelo de regresión múltiple - nomograma- convirtiéndose en un instrumento útil de predicción en el ámbito clínico.

El primer modelo estableció que la edad se presenta como variable independiente y predictora de la FV semántica. Un segundo modelo $-\mathrm{R}^{2} 62.70 \%-$ demostró también que los valores de los test de FV semántica y FV fonológica se muestran como variables predictoras.

Palabras clave: test de Fluidez Verbal, demencia, nomograma. 


\section{Abstract}

The increase in life expectancy has led to a progressive ageing of the population and an increase in the prevalence of neurodegenerative diseases. Brain degeneration may begin in middleaged individuals and since in the absence of neurodegenerative pathology there is a progressive loss of synapses during neurosenescence, early detection of some predemential state by means of neuropsychological Verbal Fluidity (VF) tests could prevent most cases of dementia from sporadically appearing.

The main cortical degenerative processes of presenil appearance affect specific cerebral areas causing linguistic alterations such as semantic aphasia linked with the integrity of the temporal lobes- or phonological -linked with the integrity of the frontal lobes-. VF declines with age, but it is not known exactly if the deterioration patterns are qualitatively different from those observed in the normal aging process.

The sample consisted of 118 subjects: $34.7 \%$ men and $65.3 \%$ women, with an average age of $52.95 \pm 18.87$. We analyzed the relationship of age with semantic and phonological VF tests, and the relationship of VF tests to each other. Thus, a multiple regression model -nomogram- was established, becoming a useful prediction instrument in the clinical setting.

The first model established that age is presented as an independent and predictive variable of semantic VF. A second model - $\mathrm{R}^{2} 62.70 \%$ - also demonstrated that the values of the semantic VF and phonological VF tests are shown as predictor variables.

Keywords: Verbal Fluidity test, dementia, nomogram. 


\section{INTRODUCCIÓN}

El incremento en la esperanza de vida ha supuesto el envejecimiento progresivo de la población y el consecuente aumento en la prevalencia de enfermedades neurodegenerativas o demencia$\mathbf{s}^{1,2}$. La degeneración cerebral puede iniciarse en individuos de mediana edad y dado que en ausencia de patología neurodegenerativa existe una pérdida progresiva de sinapsis durante el envejecimiento ${ }^{3}$, la detección precoz de algún tipo de demencia o estado predemencial por medio de test neuropsicológicos de fluidez verbal (FV) podría evitar que la mayoría de los casos de demencias fueran de aparición esporádica4,5. Las demencias carecen de medidas curativas en su mayoría, pero detectándolas en fases prodrómicas, los profesionales sanitarios podrían actuar a tiempo para prevenir o ralentizar la evolución hacia la cronicidad6. Los principales procesos degenerativos corticales de aparición presenil afectan a áreas cerebrales específicas originando alteraciones lingüísticas como la afasia semántica - vinculada con la integridad de los lóbulos temporales - o la afasia fonológica vinculada con la integridad de los lóbulos frontales ${ }^{7}-$. Sin embargo, también podemos encontrar procesos neuropatológicos vinculados con demencias subcorticales que, entre sus síntomas, incluirían trastornos motores y del lenguaje ${ }^{8}$. Por otro lado, se ha sugerido que la FV declina con la edad, no conociéndose con exactitud si los patrones de cambio/deterioro en presencia de demencia son cualitativamente diferentes de los observados en el proceso de envejecimiento normal. Los profesionales de la Enfermería tienen un papel muy importante pues, cuando sospechan o detectan síntomas neuro psiquiátricos de las demencias $u$ otros trastornos subyacentes, alertan sobre la presencia de estas alteraciones y así se establece un abordaje precoz enfocado a retrasar el comienzo del deterioro clínico y funcional ${ }^{9}$.

\section{PRUEBAS DE FV}

Encontramos cuatro tipos de pruebas de FV: categoriales, gramaticales, fonológicas y combinadas. Se caracterizan todas ellas por tener un tipo de palabras posibles capaces de ser evocadas, en un determinado espacio de tiempo y con unas reglas particulares que definen lo que no se puede realizar durante el período de evocación ${ }^{6},{ }^{10}$. El substrato neurofisiológico subyacente que se asocia con estos test se relaciona con diferentes regiones cerebrales tales como; la región fronto-temporal de la corteza cerebral - pruebas categoriales - y, en contraposición, las regiones frontales - pruebas fonológicas $-{ }^{11}$. 
Categoriales. Se vinculan con la integridad de los lóbulos temporales ${ }^{12}$ y otras áreas posteriores. Atienden al significado de las palabras durante su evocación ${ }^{13}$. Están influenciados por variables sociodemográficas como la edad o el género, aunque ciertas categorías semánticas como «nombres de personas" parecen encontrarse libres de influencias socioeducativas. La memoria semántica en pacientes con Enfermedad de Alzheimer (EA) presenta más déficits en comparación con otros individuos sanos también ancianos ${ }^{14,15}$.

Entendemos la memoria semántica como aquella que nos permite comprender el significado de las palabras, objetos y eventos, así como también nombrar y producir declaraciones significativas: así pues, la anomia es característica del deterioro semántico ${ }^{16}$. En el test de FV semántica (FVS), la emisión de diez palabras es casi cuatro veces más probable en ancianos sin deterioro frente a ancianos con deterioro cognitivo. Por ello, el test de FVS debería ser incluido en aquellos casos en que se realiza un cribado para el diagnóstico de deterioro cognitivo en el anciano que no sufre de enfermedades relevantes o limitaciones físicas o psíquicas $\mathrm{y}$, ausencia de déficits motores y sensoriales ${ }^{17}$.

Gramaticales. La gramática expresa las reglas de producción del len- guaje humano. En estas pruebas se solicitaría la evocación de palabras con una condición gramatical específica -verbos, sustantivos, adjetivos, adverbios, artículos, entre otras-.

Fonológicas. Se vinculan con la integridad de los lóbulos frontales. Atienden a los fonemas que componen una palabra. Ejemplo: solicitamos a la persona que verbalice palabras que comiencen -FV de letra inicial - o finalicen FV de letra final. También que no contengan una determinada letra -letra excluida - o bien, una cantidad exacta de letras. Se produce un menor número de palabras en pruebas fonológicas en comparación con semánticas, lo que en definitiva, nos lleva a pensar en una mayor complejidad de la tarea fonológica. De igual forma, se aprecia una mayor homogeneidad en la ejecución de los grupos con enfermedad neurodegenerativa y deterioro cognitivo leve en la condición fonológica, lo que puede traducirse en que en el proceso de envejecimiento normal, también se ven comprometidas las capacidades para la producción fluida por evocación fonológica.

Uno de los primeros síntomas en sujetos con EA es la dificultad para encontrar y/o recuperar palabras junto con una disminución de la disponibilidad léxica categorial. Por ello, la prueba de FVS es un instrumento altamente predictivo 
de un deterioro incipiente. En cuanto al punto de vista estadístico, observamos que personas sanas obtienen resultados diferentes en comparación con otros individuos con EA, así como también, hay diferencias en cuanto a los resultados estadísticos del test de FV entre EA en fase leve y moderada ${ }^{18}$. Combinadas. Demandan aspectos categoriales, gramaticales o fonológicos conjuntamente ${ }^{19}$.

El objetivo general del presente estudio de investigación fue analizar la relación del resultado de distintas pruebas de Fluidez Verbal, en un grupo de sujetos, con otras variables de interés neuroclínico.

\section{MATERIAL y MÉTOdo}

Se trata de un estudio observacional descriptivo de casos. La población diana la componían residentes de la población de Cheste de Valencia, que fuesen mayores de edad. Los sujetos se seleccionaron por un muestreo no aleatorio de conveniencia. La muestra la componían 118 sujetos a los que se les aplicó los test y se les solicitó información de tipo socio demográfica.

La selección de los sujetos se realizó a partir de criterios explícitos, objetivables, establecidos con claridad y reproducibles por otros investigadores. Los criterios de inclusión fueron: personas mayores de edad, de ambos sexos, que no presentasen ningún tipo de demencia conocida, que leyesen y firmasen el consentimiento informado, y con características geográficas y temporales accesibles a los investigadores. Los criterios de exclusión fueron: personas que no deseasen participar en el estudio, y que habiendo iniciado la contestación a los cuestionarios y por su situación de salud no pudiesen continuar.

Instrumentos. Para la recogida de datos se realizó un cuestionario ad hoc que recogía aquella información sociodemográfica de interés para el estudio, otras variables que hacían referencia el estado y hábitos de salud de los participantes, junto a los diferentes test de FV de las variantes semántica y fonológica. En el encabezado del documento se colocó información para el sujeto: identificación de los investigadores, objetivos y objeto del estudio, y agradecimientos para los participantes.

Procedimiento. Previa cita, la investigadora acudía al domicilio del participante y tras aceptarse el consentimiento informado - por escrito y de forma expresa-, se realizaba en primer lugar los diferentes test de FV, a continuación las cuestiones relacionadas con hábitos y situación de salud, y finalmente las cuestiones de tipo socio demográfico.

Tiempo de estudio. El tiempo promedio para la obtención de las (118) 
encuestas fue de tres meses. Para ello, cuando se elaboró un cronograma que contó con este espacio temporal para la obtención de todos los datos: el tiempo mínimo para cada encuesta fue de diez minutos.

El tratamiento estadístico para el análisis de los datos se basó en un análisis univariable: con las cualitativas se determinó sus frecuencias en una tabla de contingencia con su respectivo gráfico. Para las cuantitativas se realizaron medidas de tendencia central, dispersión y posición. Para el análisis bivariable se tuvo en cuenta - para establecer la relación estadísticamente significativa -un índice de confianza del 95\% para un $\mathrm{p}$ valor $<0.05$. Se determinaron constructos de interés para la investigación y sus estadísticos correspondientes.

La investigación no ha presentado conflicto de interés con persona física, empresa o institución - salvo error u omisión-. La utilización de información de otros autores en el presente trabajo de investigación, referenciada o citada, especialmente en el marco teórico y discusión, ha sido incluida en el listado de referencias bibliográficas con el estilo Vancouver ${ }^{\circledR}$.

Con relación a las consideraciones éticas y legales, la investigación se ha realizado teniendo como pilares básicos los diferentes códigos éticos y nor- mativas legales de toda investigación con seres humanos. Para el consentimiento informado la Ley 1/ 2003 de la Generalitat Valenciana sobre Derechos e Información al paciente de la Comunidad Valenciana. Los datos se trataran de forma confidencial con arreglo a la Ley Orgánica 15/1999, de 13 de diciembre, de protección de datos de carácter general.

\section{RESULTADOS}

La muestra estaba compuesta por 118 sujetos siendo el $34.7 \%$ hombres y el $65.3 \%$ mujeres, el $61 \%$ de los participantes afirmó estar casado y un $27.1 \%$ solteros. El $76.3 \%$ de los sujetos residía en área rural frente al $23.7 \%$ que habitaba en la ciudad. El $62.7 \%$ de los participantes afirmó tener estudios básicos y el $7.6 \%$ tenían estudios superiores. La media de edad de los sujetos/participantes fue de $52.95 \pm 18.87$. El participante más joven tenía 18 años frente al más mayor con 91 años.

Con relación a las puntuaciones medias de los diferentes test de fluidez verbal se presentan a continuación en una tabla con otras medidas de tendencia central, asimetría y posición (Tabla $n^{\circ}$ 1).

Análisis de predicción entre variables 
Dos modelos de regresión $-\mathrm{el}$ primero lineal y el segundo múltiple como nomograma - se ha podido realizar con $\mathrm{p}$ valores significativos, IC del 95\% y porcentajes de predicción aceptables.

\section{Modelo lineal: Regresión $n^{\circ} 1$}

En primer lugar aplicamos el estadístico Correlación de Pearson en el constructo Edad vs FV, obteniéndose un coeficiente de correlación (r) de - 0.540 (de carácter fuerte) con un $\mathrm{p}$ valor de 0.000. Así, conforme aumenta la edad, el valor en el grupo global de la prueba de FV disminuye. Los residuales que se nos presentan son -14.8890 (min), -4.9351(1Q), 0.0608 (mediana), 4.1563(3Q) y 19.6563 (máx). El Residual standard error:6.467 con 116 grados de libertad, Múltiple R-squared:0.292, Adjusted R-squared: 0.2859 y F-statistic: 47.84 en 1 y 116 DF. El modelo de predicción fue: $\mathrm{FV}=32.27$ - edad * 0.21 para un $\mathrm{p}$ valor de 0.000 y una predicción del 29\%: el valor en el test de FV disminuirá 0.21 puntos por cada año de edad (Gráfico $\left.n^{\circ} 1\right)$.

\section{Regresión Múltiple: Modelo $\mathrm{n}^{\circ}$} 2

Se planteó valorar y analizar cómo se relacionaban las diferentes pruebas de FV y cómo el valor de FV FO ER y FV SE $P$ predice el valor de la prueba de FV A. Así, la variable depen- diente será FV A y como variables independientes: FV FO ER y FV SE P. Resumen del modelo. El resultado del análisis nos ofrece una $R=0.792$, una $R$ cuadrado ajustado de 0.620 y un error estándar de la estimación de 4.665. El resultado de los coeficientes es el siguiente: para la variable constante se obtiene una coeficiente no estandarizado de 6.125 con un error estándar e 1,298, para FV SE P 0.320 con 0.066 de error y para FVFO R 0.930 con 0.135 de error. Todas las variables con una significación de 0.000 .

El modelo de predicción será: FV SEM A $=6.125+$ FV SEP ${ }^{\star} 0.32+$ FV FO $E R{ }^{*} 0.93$. Para un $p$ valor de 0.000 y una predicción del $62.70 \%$. Tal y como se muestra en la fórmula de predicción, el valor en el test de FV SEM A aumenta 0.32 puntos por cada respuesta en el test de FV SE P y, 0.93 puntos por casa respuesta de FV FO ER.

Podemos afirmar que, obtenido un $\mathrm{p}$ valor $<0.05$ y con una predicción del $62.70 \%$, los valores de los test de FV SE P y FV FO ER se muestran como variables predictoras del valor de FV SEM A.

\section{Discusión Y CONCLUSIONES}

En el modelo de regresión $n^{\circ} 1$ se obtuvo que la edad se presenta como variable independiente $\mathrm{y}$, en este caso, 
predictora de la FV semántica. La evidencia científica al respecto nos indica que, a medida que aumenta la edad, disminuyen diferentes capacidades cognitivas, no solamente en lo que respecta al deterioro cognitivo leve (DCL), sino también, en diferentes enfermedades neurodegenerativas como la EA - demencia más prevalente- produciéndose una disminución en la producción de palabras. Así pues, es preciso valorar cómo influyen el resto de pruebas de FV sobre la FV semántica17.

Un segundo modelo de regresión y, con una predicción del $62.70 \%$ demostró que los valores de los test de FV semántica de palabras y FV fonológica ER se muestran como variables predictoras del valor de FV semántica - de animales-, conformando de esta forma un nomograma de predicción.

A través de las pruebas de FV - semántica y fonológica- se ha podido correlacionar la puntuación de cada una de ellas con las áreas cerebrales asociadas con el lenguaje ${ }^{16}$, especialmente el área de Broca y Wernicke -funciones de expresión y comprensión-. Otras variables dieron como resultado que, en el grupo global, seis de cada diez sujetos afirmaron tener alguna enfermedad. A su vez, también se hace preciso resaltar que la ingesta diaria de número de fármacos obtenida en la muestra a estudio era de 2.01 \pm 3.22 . En las pruebas de contraste de hipótesis en busca de relaciones estadísticamente significativas, en concreto con el constructo de edad y sexo y otras variables de interés, no se han obtenido valores significativos. En lo que respecta a la contestación de los sujetos a variables dependientes que pudiesen afectar a las pruebas de FV y, especialmente a la comorbilidad, dos de cada tres participantes afirmaron tener alguna enfermedad: la evidencia nos indica que conforme aumenta la edad, también lo hace el número de enfermedades ${ }^{19,20}$.

Continuando con la inferencia entre género y las respuestas a los test de FV, no se ha encontrado significación estadística en las diferentes pruebas de FV semántica. Sin embargo, en un análisis descriptivo se observa cómo el $p$ valor aumenta su valor, pudiendo afirmarse que, conforme aumenta la dificultad en las diferentes pruebas, se obtiene una disminución en la significación.

La edad, como variable cuantitativa, ha correlacionado con los diferentes test de FV mostrando resultados concordantes con la realidad biológica en el sentido de que, un aumento de la edad, disminuye la capacidad cognitiva relacionada con el lenguaje.

Así, la edad junto a la carga genética se muestran como factores no 
modificables. En la medida que podemos actuar sobre los modificables, como por ejemplo el ejercicio físico y el cognitivo, seremos capaces de ralentizar los efectos deletéreos que produce el paso de los años, tal y como afirman diversos autores ${ }^{17,19}$.

Con relación a las puntuaciones de las diferentes pruebas de FV, se observó una disminución de las puntuaciones medias conforme iba aumentando la dificultad de las mismas en el test objeto de trabajo: de las pruebas de FV semántica a las pruebas de FV fonológica disminuye el rendimiento, ya que en estas últimas, es necesario conectar más de una zona/área cerebral. Igualmente, todos los índices de correlación (r) resultaron negativos para los constructos Edad frente a las diferentes pruebas de FV - por lo que, a medida que aumenta la edad disminuye la capacidad en el lenguaje. En la demencia asociada con la edad, existe una reducción de la producción de palabras tanto en la modalidad fonética como semántica12.

\section{Futuras líneas de investigación}

Planteamos la necesidad de que se desarrolle una mayor implicación de Enfermería en el campo de la investigación. Especialmente, en el de la detección de neuropatologías por medio de test neuropsicológicos ya que, dadas sus competencias como profesionales sanitarios de la salud, son los encargados de llevar a cabo estas actividades. 


\section{BiBLIOGRAFÍA}

1 Rommy M. Envejecimiento: cambios bioquímicos y funcionales del Sistema Nervioso Central. Rev Chil Neuro-Psiquiat. 2005;43(4):297-304.

2 Rodríguez A. Factores de riesgo cardiovascular y deterioro cognitivo. Rev alzheimer.

2007;36:30-37.

3 Matthews F, Brayne C, Lowe J, McKeith I, Wharton S, Ince P. Epidemiological pathology of dementia: Attributable-Risks at death in the medical research council cognitive function and ageing study. Rev PLoS Med. 2009;6(11):1-15.

4 Tortora J, Derryckson B. Principios de anatomía y fisiología. 13a edición. Madrid: Editorial médica panamericana;2013.

5 Hogan D, Bailey P, Black S, Carswel A, Chertkow $\mathrm{H}$, Clarke B, Cohen C, Fisk J, Forbes D, Man-SonHing M, Lanctôt K, Morgan D, Thorpe L. Diagnosis and treatment of dementia: 5. Nonpharmacologic and pharmacologic therapy for mild to moderate dementia. CMAJ. 2008;179(10):1019-1026.

6 López A., Calero M, Navarro-González E. Predicción del deterioro cognitivo en ancianos mediante el análisis del rendimiento en fluidez verbal y en atención sostenida. Rev Neurol. 2013;56(1):1 7 Montañés P, Matallana D, García R, Cano C. Deterioro selectivo del lenguaje debido a degeneración temporal focal: análisis comparativo entre un caso de afasia primaria progresiva y un caso de demencia semántica. Rev Asociación colombiana de gerontología y geriatría. 2001;42(3):119-130.

8 Galeote M, Peraita H. Memoria semántica y fluidez verbal en demencias. Rev Esp de Neuropsicología.1999;1(2):3-17.

9 Persoon A, Joosten-Weyn B, Van de Vrie W, Olde M, Van Achterberg T. Development of the Nurses' Observation Scale for Cognitive Abilities (NOSCA). ISRN Nursing. 2011; doi: 10.5402/2011/895082.

10 Fernández A, Marino J, Alderete A. Valores normativos en la prueba de Fluidez Verbal-Animales sobre una muestra de 251 adultos argentinos. Rev Argentina de Neuropsicología. 2004;4:12-22.
11 Ferreira D. Fluidez Verbal en el Deterioro Cognitivo Ligero: Análisis cuantitativo y

cualitativo. Tesis Doctoral. Facultad de Psicología. Universidad de la Laguna: Tenerife. 2013.

12 Marino J, Alderete A. Valores normativos de pruebas de fluidez verbal categoriales, fonológicas, gramaticales y combinadas y análisis comparativo de la capacidad de iniciación. Rev neuropsicología, neuropsiquiatría y neurociencias. 2010;10(1):79-93.

13 Sailor K, Antoine M, Diaz M, Kluger Al, Kuslansky $G$. The effects of Alzheimer's disease on item output in verbal fluency tasks. Neuropsychology. 2004;18(2):306-314.

14 Rogers T, Ivanoiu A, Patterson K, Hodges J. Semantic memory in Alzheimer's disease and the frontotemporal dementias: a longitudinal study of 236 patients. Neuropsychology. 2006;20(3): 319-335.

15 López A, Calero M, Navarro-González E. Predicción del deterioro cognitivo en ancianos mediante el análisis del rendimiento en fluidez verbal y en atención sostenida. Rev Neurol. 2013;56(1):116 Peraita H, González MJ, Sánchez ML, Galeote MA. Batería de evaluación del deterioro de la memoria semántica en Alzheimer. Psicothema. 2000;12(2):192-200.

17 Carmona-Simarro JV. Análisis de factores psicosociales en pacientes con enfermedad de Alzheimer: tarifa social, salud percibida y calidad de vida. Tesis Doctoral. Universidad CEU Cardenal Herrera: Valencia.2012.

18 Baldo JV, Schwartz S, Wilkins D, Dronkers NF. Papel de la corteza frontal contra temporales en la fluidez verbal según lo revelado por Voxel confección de mapas de los síntomas lesión". J Int Neuropsychol Soc, 2006;12(6):896-900.

19 Mc Dowd J, Hoffman I, Rozek E, lyons K, Pahwa R, Burns J, Kemper S. Understanding verbal fluency in healthy aging, Alzheimer's disease, and Parkinson's disease. Neuropsychology. 2011;25(2):210-225.

20 Instituto Nacional de Estadística (INE). Disponible en: www.ine.es [Visita 21/7/2018]. 


\section{TABLAS Y ANEXOS}

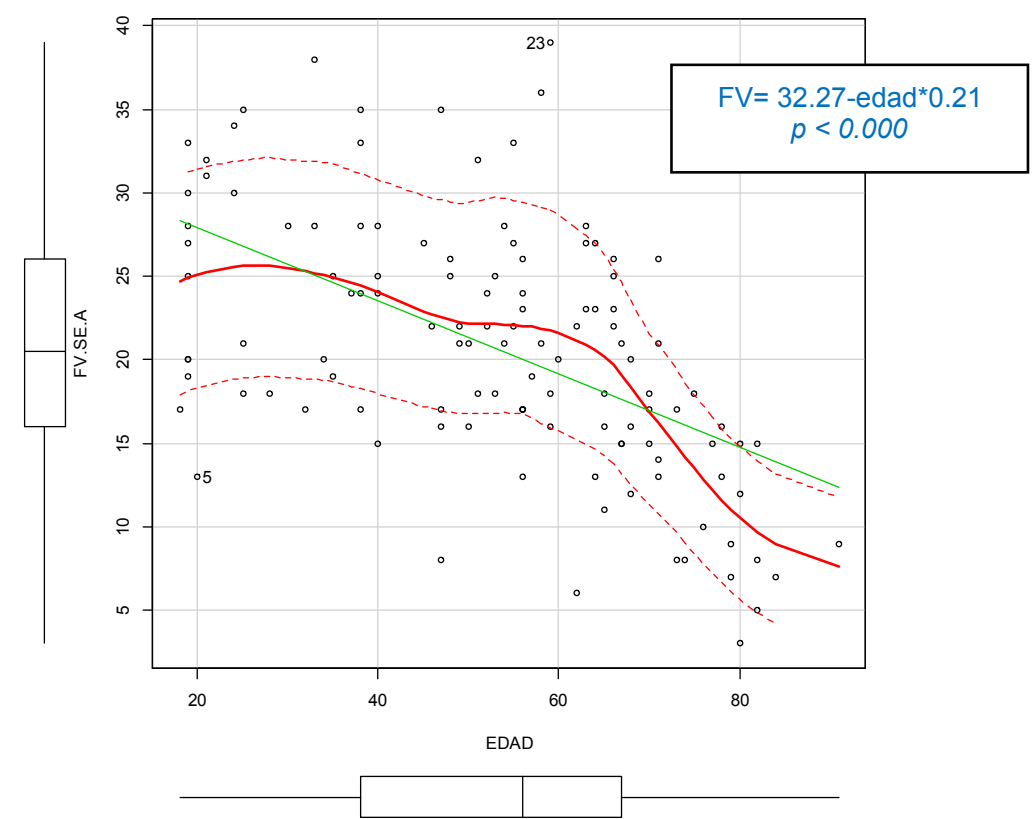

Figura $n^{\circ} 1$ Recta de regresión. Diagrama de dispersión «Edad vs FV» 


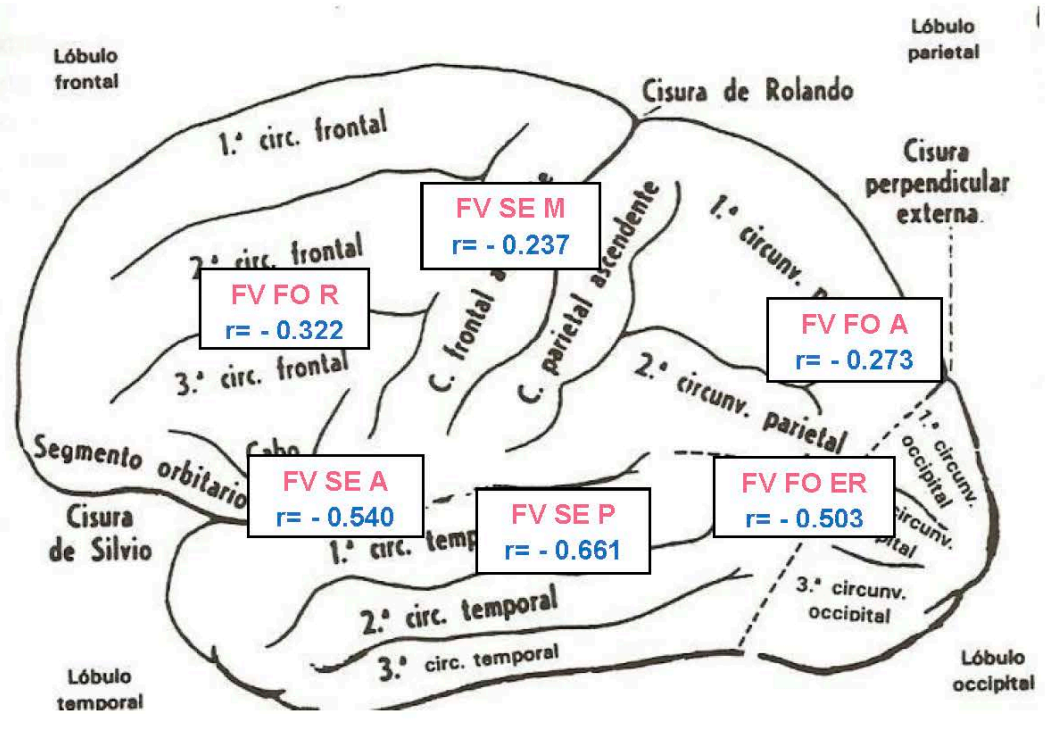

Figura $\mathrm{n}^{\circ}$ 2. Coeficiente de correlación de los distintos test de FV 